\title{
The Hanoak House as a Flexible and Adaptable Vernacular Precedent for Modern Architecture
}

\author{
Jan M. Hugo \\ Department of Architecture, University of Pretoria, Pretoria 0028, South Africa
}

Corresponding Author Email: jan.hugo@up.ac.za

https://doi.org/10.18280/ijsdp.160413

Received: 23 May 2020

Accepted: 18 March 2021

\section{Keywords:}

vernacular architecture, flexible adaptable design, climate change adaptation, Korean architecture

\begin{abstract}
Globally the adverse effects of climate change necessitate the implementation of resilient systems that respond to escalating weather fluctuations and increased urban vulnerability. This requires a shift from the traditional efficiency-focused solutions, towards robust, responsive and flexible models. While novel technologies are being developed to address these needs; existing vernacular examples also present innovative solutions. The purpose of this study is to analyse vernacular solutions, in this case Korean Hanoak housing typologies, in terms their integration of flexible and adaptable spatial and technological systems to inform modern applications. As research method, the study firstly employed an unstructured observational method to document the spatial and technological elements of these vernacular precedents, followed by an intersubjective literature review of these precedents to understand the historic context. As main conclusion the study identified seven design principles to inform the development of flexible and adaptable modern architecture solutions. These include: holistic, integrative design; articulated and reciprocally layered systems; nested levels of flexible and inflexible systems; appropriate scale identification; and appropriate technology use. As contribution, this article analyses existing vernacular precedents and highlights principles that can be applied in various contexts to develop locally responsive and flexible architecture.
\end{abstract}

\section{INTRODUCTION}

Achieving homeostasis within a changing and fluctuating environment, has been a continual challenge for humankind. While in some cases achieving thermal comfort is considered a luxury, in extreme climatic conditions this is not always the case. Within severe climatic conditions the ability to modulate the indoor climate is considered critical to ensure survival.

The practice of achieving a comfortable indoor environment developed over several millennia, resulting in numerous vernacular typologies [1]. These building solutions have been developed over a long period and incorporate the climate, local geographic conditions, available local materials and technologies, socio-cultural, and economic factors in the vernacular typology generation [2]. While these typologies developed within a continuity of stable climatic conditions and consistent material availability, in future these stable conditions are not guaranteed.

Climate change is set to bring about rapid and severe changes to both rural and urban conditions [3]. These changes, brought about by global warming will result in a series of heterogeneous impacts that must be dealt with on a local basis within individual communities [4]. One such approach would be to thoroughly understand the impending impacts and prepare for the impacts with exact measures. Yet, as noted by Walker and Salt [5], this often results in efficiency-focussed solutions that in effect increases the vulnerability of the system. An alternative approach is to develop building solutions that adapt to a range of impacts - increasing their flexibility and adaptability to changes and impacts [5]. This requires achieving such levels of flexibility and adaptability within the built environment on various scales.

While calls for alternative designs and strategies in the built environment, and their associated solutions, are not necessarily new. Pressure to adjust our cities and built environment in response to long term climate changes as well daily weather fluctuations are mounting [3,6]. These forms of adaptability and open building concepts are not restricted to building services but also the programming, giving rise to multi-functional, multi-programmed spatial arrangements [7, 8]. This is specifically important on smaller, more affordable scales in future cities and housing. While solutions can be found in new technology and systems; vernacular precedents can also be informative and instructive in finding current-day solutions [9]. Developing the architectural discourse on flexible and adaptable architecture by considering existing vernacular responses can reveal strategies and approaches that are more accessible for implementation in the everyday context.

In summary, there is an increasing awareness of rapidly changing conditions within which static (homeostatic-biased) solutions are inappropriate. While an increasing number of academics and practitioners are calling for flexible and adaptable architectural solutions, often expressing solutions based on novel technologies and materials, historic or vernacular solutions can be of similar value. As a result, this study undertakes a descriptive analysis of flexible and adaptable Hanoak precedents located in South Korea. This article sets out to analyse existing vernacular precedents, to define spatial and technological principles that can inform 
modern architectural applications. While limited literature on the flexibility of Hanoak architecture is available, this study builds on the work of Kim and Jia [10] by adding to their identified flexible aspects and generalising these into principles for implementation in modern alternative architectural solutions. This study, therefore, sets out to improve our understanding of adaptable and flexible architecture and how this can be achieved using simple locally responsive architectural solutions.

The article is structured along three sections. It commences with the literature review and theoretical argument that highlights the need for adaptable and flexible architecture. The next section defines the method used to document the precedents, followed with a discussion of the architectural elements and their role within a flexible framework. The final discussion and conclusion define a set of principles to achieve flexibility through the spatial and technological articulation of architectural solutions.

\section{TOWARDS IMPROVED FLEXIBILITY ADAPTABILITY IN CHANGING CONDITIONS}

AND

While climate change affected disruptions can be expected on a global scale [3], these negative impacts and changes often play out in local contexts in diverse ways. As the relationship between the inputs driving climate change and the resultant impacts are often removed by both space and time [6], the difficulty in accurately predicting the changes makes preparation for these impacts increasingly complex.

The global climate system will generally shift towards climatic conditions with higher thermal energy contained in the system [11]. Resulting in cascading adverse impacts in multiple regions globally [12]. While a general loss in favourable stable conditions is expected, these changes will also result in significant levels of extreme weather events and changes in local climates. As a result, weather events that are higher in intensity, frequency and amplitude can be expected. Several studies considered macro-climatic changes on regional levels, yet on a smaller microclimatic scale the actual conditions of these climatic changes are more varied and can be only be predicted with limited accuracy [11]. While there are a number of studies advocating developing tools to test and simulate extreme weather conditions [13], more development is still needed in that field. As a result, designers in the built environment are, and should be, less confident of expected weather conditions, and can with certainty expect extreme changes in the weather patterns.

While building designers do not necessarily know what the exact impacts or changes will be, it is certain that dramatic weather changes will become the norm. Responding to specific conditions will be difficult, if not impossible. Within this context of rapid and extreme changes, cities often develop within a paradigm of continued stability and the built environment is slow to adapt to sudden changes. Furthermore, the exposure and limited adaptive capacity of the built environment often further accentuate their vulnerability [14]. While limiting the exposure of the built environment to climate change induced hazards is possible in newly built scenarios, many of our cities and existing built environments are already constructed and will require retrofitting as climate change adaptation responses. Many advocate improving local adaptive capacities as viable responses to limit the exposure of the built environment and users to potential adverse impacts
$[15,16]$. These include, increased local response capacity, effective and fast feedback systems, access to relevant and appropriate technological solutions, and developing effective and quick measures to sudden and distinct changes $[15,17]$.

A potential response measure, to the uncertainty coupled with disruptive changes, is to focus on flexibility, adaptive responses, and our capacity to learn and innovate [18]. This requires responses that are more flexible, rather than further investing in hard, robust and impenetrable solutions. While designers often simply respond to the existing context as a stable continuity leading to inappropriate existing built solutions; existing vernacular examples can still provide insight into alternative means of design and construction [19]. As noted by Crichton et al. [6], many vernacular precedents have survived changes, be it social, environmental or economic, for a number of years. Crichton et al. [6] continue identifying levels of flexibility ranging from nomadic lifestyles to adding or removing thermal energy from the structure itself. As key argument this study proposes that vernacular examples can be present current designers with models of flexibility that will critical to consider when designing or retrofitting our current built environment in the wake of climate change disruptions.

\section{FLEXIBILITY AS FORM OF ADAPTIVE CAPACITY TO ACHIEVE CLIMATE RESILIENCE}

Climate change adaptation can be considered as a means towards alternative, resilient cities and living conditions. As noted by the IPCC (2014a) adaptation is defined as "... [the] adjustment [of natural or human systems] to actual or expected climate and its effects... to moderate or avoid harm or exploit beneficial opportunities". Adaptation therefore aims to both limit the adverse effects of climate change, but also hopes to exploit any beneficial outcomes from these impacts. O'Brien and O'Keefe [15] argue that climate change adaptation must be not be considered as the end, but rather the means to achieve resilience.

Dipasquale et al. [19] identify resilience embedded within vernacular architectural forms, as slow yet continually dynamic evolution of locally responsive solutions often retaining resilient characteristics. Dipasquale et al. [19] continue stating that these characteristics include sharing of knowledge imbued within the built form and local traditional knowledge; furthermore that self-built nature allows for rapid post-disaster recovery, and being highly integrated with context and site often allow for sustainable responses that are receptive to change. While the resilience discourse developed significantly and vary in its definition, a study by Folke et al. [20] argue for embedded flexibility and adaptability in resilient responses rather than focussing on robust, rigid solutions.

Flexibility can adjust lower local climate change risk levels. As local risk levels are affected by the inhabitants' sensitivity to the risk, exposure to the hazards, and the scale of the hazards themselves; the flexibility to adjust the adaptive capacity of the community or entity can limit both the local sensitivity and exposure [21]. Heinrichs et al. [22] call for solutions that improve the capacity of individuals' to respond to extreme disruptions, and also change or adjust their environment in anticipation of disruptions or in the aftermath of these changes. While adaptive capacity is widely promoted in studies considering climate change adaptation and its application in 
multiple contexts $[15,16,23,24]$, the relationship between flexibility and adaptive capacity still remains to be considered. This study postulates that in order to effectively respond to the climate change induced impacts, a flexible built environment is needed.

Flexibility must be considered on multiple scales. While cities can be defined as massively scaled entities resulting in significant inflexibility as a whole, Cartwright et al. [25] argue that these very cities are continually in flux and change. It is therefore the change and flux of smaller entities that increase the adaptive capacity of the whole. Yet, the nature and scale of these strategies must be considered, Carter [23] identifies several levels at which adaptive strategies adjust the urban environment - one of these being the architectural scale.

This level of architectural flexibility takes place in multiple forms as Till and Schneider [8] argue that there are a number of soft strategies, indeterminate in nature, and hard strategies, more determinate in technology and spatial articulation, to achieving it. Sung-Hwa and Beisi [10] similarly identify standardisation and regulation, construction practices, and technological systems in Hanoak houses contributing to their flexibility in use and application. Tan [7] undertook a study of Ancestral halls in China, and identifies the long term adjustable reuse of the architectural artefacts as important modes of flexibility. Tan [7] continues arguing that this level of flexibility is facilitated in the spatial and material articulation of the architecture itself. Crichton et al. [6] argue that many historic societies have managed to adjust to disruptive changes. One of the principle means to ensure such survival is ensuring flexibility to change, Crichton et al. [6] continue identifying a series of levels of flexibility that include:

- mobile structures or cities,

- adjusting form or material of structures,

- $\quad$ using different parts of the structure as the seasons change,

- adding additional energy to ensure comfortable indoor environments,

- $\quad$ adjusting lifestyles to these changes.

If designers of the built environment aim to ensure the long terms viability of these spaces, adaptive capacity must be promoted. While adaptive capacity has multiple facets, it is important to consider the ability of users to adjust their own spaces according to changes experienced and in anticipation to significant disruptions [17]. This requires rapid flexibility to adjust the built environment while it is in use. While modern projects, such as Terrence Donnelly centre in Toronto or the Al Bahar Towers in Abu Dhabi [26], use innovative and novel technologies to achieve such flexibility, this study aims to consider the construction, assembly, material choice and use of a vernacular historic example as means to inform flexible strategies in the modern built environment.

\section{RESEARCH METHODS}

The study followed a qualitative, immersive approach to analyse an existing vernacular building typology. This approach required both visual observational assessment, phenomenological experiences of the place, as well as an historic analysis of the architecture itself. This process specifically focussed on the spatial, technological application of these vernacular examples.

The observational analysis followed an unstructured qualitative observational approach, during which sketches and photographic analysis of the precedents were undertaken. This allowed the researcher to document a variety of aspects, including seemingly unimportant aspects which only prove important later. In order to improve one's understanding of the spatial and technological articulation of the architectural typology, the phenomenological centred study included a literary analysis, following a more intersubjective approach, to understand both the context and product in its setting and time as continuum [27]. A literature study on existing Korean vernacular architecture, their influences and development was conducted, unfortunately limited literature is available in English adding more pertinence to this study.

While a number of these buildings and sites are deemed national heritage sites with Hanoak houses preserved in their historic context, their continued upkeep result in these buildings being effectively reproductions of their original forms. Yun [28] raises a concern regarding the level of authenticity in the exact reconstruction of the precedent, but for this study the principles of design and construction is of value. On the other hand, as these studies are not actively used, these have limited value to inquiries of the lived experiences of these buildings. To better understand these examples, the researcher also visited a number of exiting Hanoak houses that are still in use. These represent the more modern use of historic buildings and building typologies, and allowed the researcher to uncover modern adaptations of these building types.

The sample selection includes several residential precedents originating from the Joseon period in the Korean peninsula. The sample selection is located in South Korea, and are all deemed exemplary residential examples of the Joseon period. All precedents share a series of common technological elements, systems and spatial approaches. The sites that were visited included:

a) The private residences of the Joseon Dynasty in Gyeongbukung and Chandeokung, Seoul, South Korea.

b) Yangdong Village, Gyeongju, South Korea.

c) Hwangnamdong, Gyeongju, South Korea.

d) The Hanoak House museum, Paju Book city, Paju, South Korea.

\section{CONTEXT, CULTURE AND CLIMATE AS DESIGN INFORMANTS}

The Hanoak house as vernacular residential typology, developed mainly outside of city centres due to the improvement of agricultural practices and technologies brought over from China's Yaun Dynasty [29]. It was generally used by the scholar upper-class, Yangbang, from the early Joseon period in the 16th century [28, 29]. Many of these houses have been used well into the 20th century, with few examples being well preserved and still occupied in suburbs in Seoul, Gyeongju and Andong in South Korea (Figure 1).

The typology originated from two divergent ideological or cultural systems. This occurred during the transition from the Goryo to Joseon Dynasty's in the 14th century, which resulted in the national religion and philosophy shifting from Buddhist to Confucian traditions [30]. As a result, the Hanoak houses presented the sparse interiors and spatial layouts, and limited decoration following Confucian principles [29, 30], while the site selection and orientation were informed by feng shui a concept brought to Korea by the Seon (Zen) Buddhist sect in the 10th century $[29,30]$. This led to architectural interventions which are highly adapted and sensitively positioned, harmonising with its natural surroundings as noted in Figure 2 [29]. 


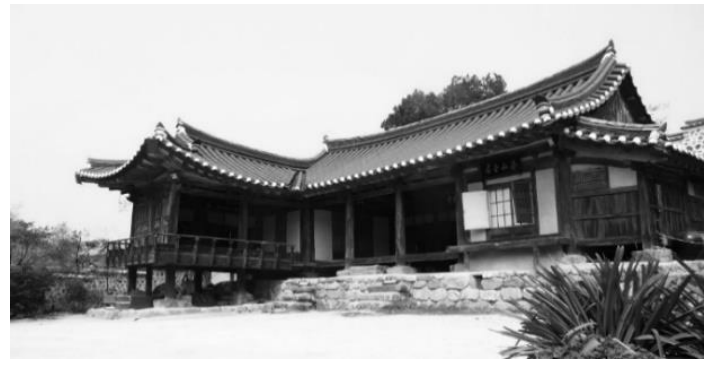

Figure 1. Macheongdong, Yangdong, Gyeongju, South Korea

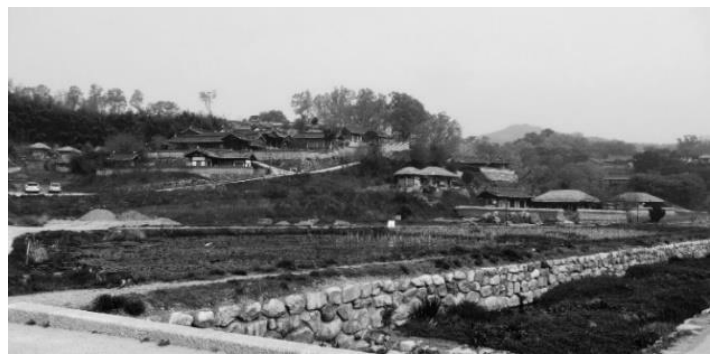

Figure 2. Yangdong Village, village layout integrated into the landscape

The highly regulated and hierarchical Confucius cultural system stipulated the use and development of various residential typologies and who may build these. All material use, decoration, structural system and sizing of the houses were controlled according to class since the Shilla period in the 7 th century $[10,29,30]$, resulting in homogenous and highly regulated townscapes, using similar local construction material. This also resulted in the prohibition of decoration of private homes since the 14th century in the Joseon era [10]. This highly regulated context resulted in the development of specific vernacular typologies.

Firstly, the highly regulated class structure controlled the size and scale of the various houses. The similar scaling of the buildings (typically no more than 10 ch'oks or 3 meters), along with the Confucius tradition of having multi-generations living in gender-specific and structured order, led to residential complexes made up of a complex series of courtyards and buildings $[29,30]$. As a result, these complexes often manifest in as several a-symmetrical buildings, unified with a single roof.

These houses mostly made use of commonly available materials, including timber and stone, while masonry was rarely used [29]. As a result, these houses were mostly lightweight timber structures with high mass stone and clay floor bases and tiled roofs [29]. Notably, tiled roofs are typically associated with precedents originally owned by the upperclass, the lower class residents used thatch as roofing materials [28].

Substantial number the buildings were constructed by Buddhist monks using the Taillang (column-beam-strut) construction methods, these technological and building methods originated from Southern China and were brought into Korean peninsula along with the introduction of Buddhism [10, 29]. While the temples and government buildings used more elaborate multibracket Taillang systems, the Hanoak houses typically used a simple column-and-beam system scaled to suit the smaller roofs.

The ban of ornamentation and decorations, and the regulated use of colour, resulted in many houses using the local materials in their natural state presenting an authenticity of material use [30]. Similarly, the timber screens, paper screens used to articulate the building envelope and handrails, often used intricate lattice work as substructure or screens [30]. Rather than focusing on ornamentation the materiality and structures themselves were celebrated.

Thirdly, the highly stratified hierarchy, along with the gender-specific roles that were articulated in the Confucian culture, led to the gender-specific rooms (sarang-ch'ae and an-ch'ae) being used as multifunctional entities [10, 30]. Similar patterns were documented in the associate courtyards (madang) related to these specific gendered spaces.

Finally, while culture and religion played a major role in the development of Hanoak architecture, climate has had a similarly paramount influence on the architectural typology [30]. The Korean peninsula is positioned on the south-eastern edge of the Eurasian continent and borders the Pacific Ocean and East China Sea. As a result, it is exposed to divergent and complex climatic conditions, ranging between hot and humid summer seasons with extreme rainfall events to long dry cold winters with heavy snowfall [31]. Extreme seasonal temperature fluctuations range from $40^{\circ} \mathrm{C}$ with $80-90 \%$ humidity, in Summer, to temperatures of $-32^{\circ} \mathrm{C}$, with an average humidity of $30-50 \%$, in Winter [31]. These diverse climatic conditions gave rise to flexible architectural responses.

As discussed, the context, climate and culture played principal roles in informing the local Korean vernacular architecture. As a result, the Hanoak typology represents a local solution developed over long periods, integrating local informants and adjusting imported technologies to the context.

\section{FINDINGS: FLEXIBLE AND ADAPTABLE BUILDING COMPONENTS}

Within this environment of climatic, social, cultural and statutory influences; emerged a housing typology that is highly flexible in its function, space utilisation, and climatic response (Figures 1 \& 3). These forms of adaptability are achieved through an interdependent array of spatial and technological elements.

\subsection{Anthropocentric scaled design}

Informed by the Confucian ideal of modesty and moderation, the typical Hanoak house is very restrained in scale, size and embellishment [29, 30]. All rooms are uniform in size and design, and relate to the overall scale ( $k^{\prime}$ an $)$ of the building, resulting in a well-scaled, anthropocentric housing typology (Figures $1 \& 3$ ). These rooms also exhibit limited use of spatial signifiers, resulting in spaces that are all temporal and interchangeable.

The typical Hanoak house is separated into gender-specific spaces [30]; each section consisting of a main hall (Teach'ong) and a series of smaller side rooms. While the main hall is commonly large enough to accommodate important rituals and daily functions [30]; the smaller rooms are typically $3,5 \times 3,5 \mathrm{~m}$ meters (ranging between two to three k'an) in size and only accommodate two to three persons at a time [29]. The main hall is specifically void of any furniture to allow for its multifunctional use.

These smaller rooms all have large sliding doors (changjimun or tukkoptajimun) and removable screens (punhapmun and pulbalgimun) linking each other and the main hall - therefore allowing the extension or separation of different rooms depending of time, season and functional 
requirement [30]. While the smaller rooms are highly flexible and open towards each other and the main hall; the main hall specifically opens up towards the landscape and the central courtyard. Resulting in a compact built form with a balanced interplay of open and closed spaces (Figure 3), articulating private and semi-public spaces on a residential scale $[29,30]$.

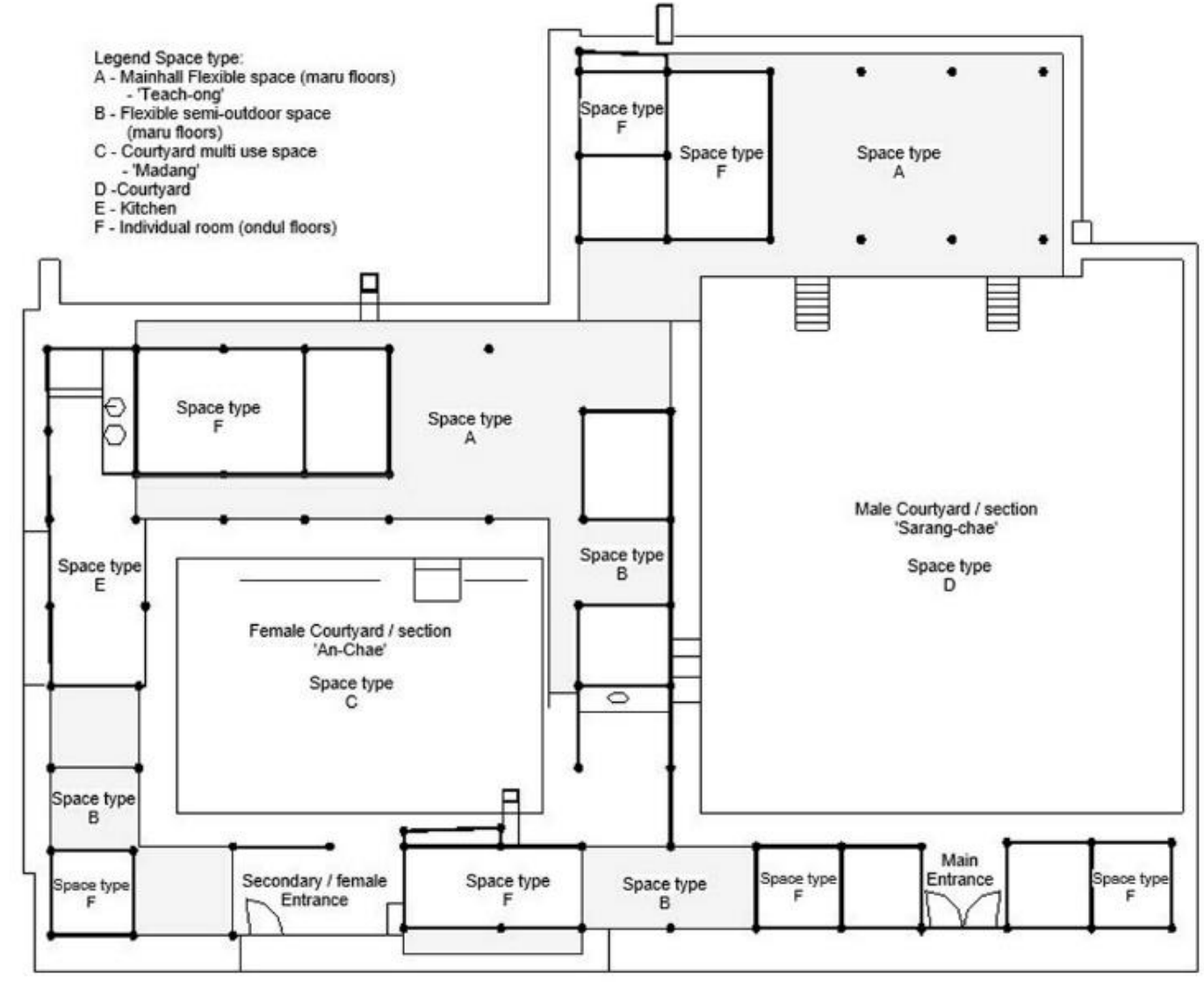

Figure 3. General layout of a Hanoak house. This example is the floor plan of Yangjin-dong, Andong, South Korea (Source: Redrawn from Kim [29], and Kim and Jia [10])

\subsection{The courtyard as fulcrum}

While various adaptations in terms of the spatial arrangements and local typologies of the Hanoak house are typically found throughout the different regions in South Korea [29], all these typologies, make use of a central courtyard (madang). The central courtyard is an integral part of the Hanoak house as spatial element, enabling multifunctional space use and added climate control (Figures $3 \&$ $4)$.

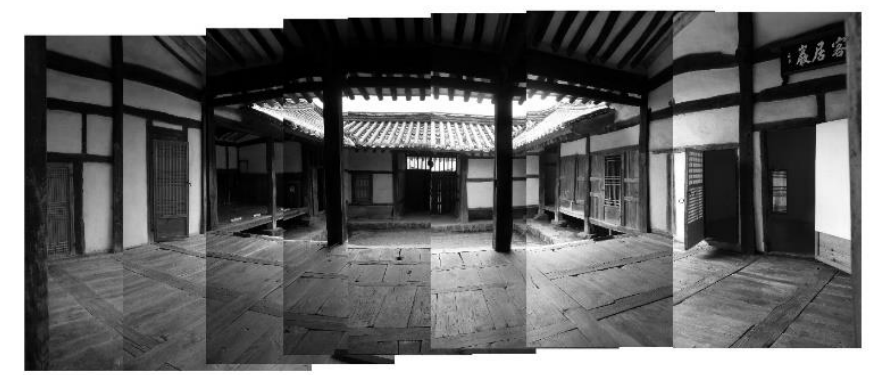

Figure 4. Courtyard of Gwang-ga-jeong, Yangdong, South Korea

The courtyard functions as a multi-functioning outdoor room accommodating work, rituals and rest [10, 29, 30]. It is usually an open space with a compacted earth floor finish, with little or no plants or gardening - leaving it therefore undefined for the variety of uses.
In more elaborate Hanoak houses a series of courtyards are used throughout the design (Figures $3 \& 4$ ). This provides an additional level of privacy for the gender-specific spaces. According to the Confucian customs of the Joseon era, the womans' quarters were positioned in the deepest part of the house; allowing only the immediate family and females to enter it [30]. The use of additional building structures, screens walls (nae'oe) and gates (chungmun) provide separation and privacy.

In terms of climate control the courtyard plays an integral part in the overall strategy. With the courtyard the building depth is minimised allowing for adequate cross ventilation during the hot, humid summer months. In the cold winter months the courtyard allows direct sunlight reaching deep into the interior spaces, simultaneously protecting these spaces from the strong northern winter winds.

\subsection{Structural system, floor and roof-framework for multifunctional use}

Using local materials-stone, timber and clay-the Hanoak house makes use of a timber column and tie-beam structure. This framed structural system, along with a robust and heavy roof, and multi-zonal floor plate incorporates an array of adaptable, multifunctional spaces that accommodates both short and long term changes.

The main structure becomes one of the basic frameworks accommodating flexible walls and planes (Figure 3). A timber column and tie-beam structural framework - scaled according to a proportional system of $k^{\prime}$ an and ch'oks $[30,32]$ allows for 
lightweight walls, planes and openings to be used as in-fill. These can be adapted and changed at different intervals and as the need arises.

The roof structure in comparison to the whole building structure seems excessive and overly complicated, yet the elaborate structure becomes multivalent in nature (Figure 5). Firstly, the gentle sloping roof acts as unifying element in the Hanoak house; accommodating irregularities encountered when modifying the building layout to the landscape and binding the whole together. Using a simplified bracket system (dapo) the roof structure is scaled to the overall proportional system [30]. Secondly, it functions as climate control element, incorporating an insulating layer of clay and tiles for the cold winter months, furthermore the steeply sloping roof also quickly dispels rain during the summer typhoon seasons.

The ceiling finish provides the final level of adaptation to the roof. Timber framed and paper covered drop-ceilings (ch'anjongs) are used in the individual rooms, to minimise the overall volume and add additional insulation for the cold winter months. While in the main hall (taech'ong) the roof is left exposed to minimise thermal retention and improve ventilation in the hot humid summer months [30].

Typically, a Hanoak house uses two different floor systems, being the ondul (heated) and maru (cooled). The layout of these two floor systems is used to articulate and reflect the Confucian principles of contemplation, scholarship and harmonising with nature, creating a tension between open (maru) and closed spaces (ondul) [29]. While also being used for indoor temperature control, the elevated floor plane also clearly articulates the threshold between the courtyard (madang) and main hall (taech'ong) - removing, therefore, the need of walls to articulate the space (Figures 1, $3 \& 4$ ).

As an elevated plane the maru becomes a low mass ventilated timber floor - providing coolth in the summer. Following the Wumulmaru construction method using tongue and groove joints the substructure and floor finish are integrated into a single plane [30].

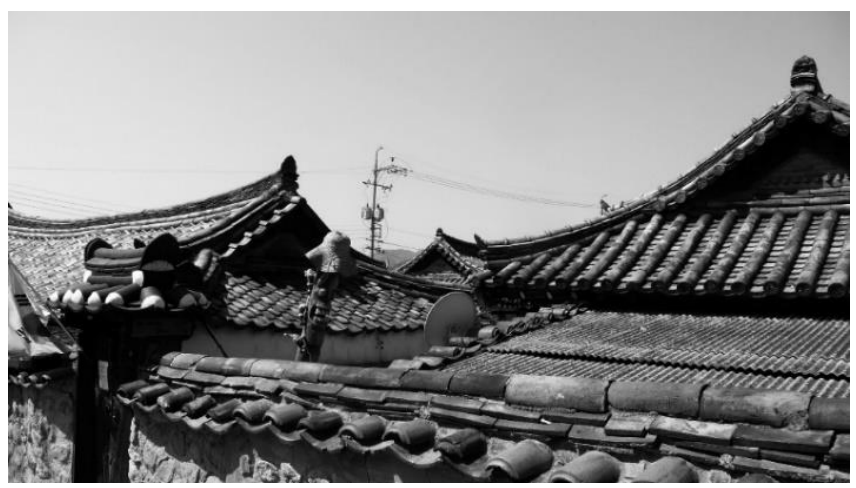

Figure 5. Unifying roofs of various Hanoak houses, Hwangnamdong, Gyeongju, South Korea

In contrast, the ondul floor is a hypocaust heated floor, using the heat generated in the kitchen stove and channelling it, using horizontal flues, through the floor space to a chimney on the opposite side [30]. This high mass clay and stone floor, finished with oiled paper, cotton or natural resins, rapidly transfers heat into the interior, effectively recycling the heating energy and efficiently storing it in the high mass floor structure [30]. This system has been used in Korean domestic architecture since the 12th century and is believed to originate from the Manchurian Kang heating system [29].

\subsection{Walls and screens as movable planes}

Completing the structural and spatial flexibility - the walls and screens merge the whole system into a functioning whole (Figures 6 \& 7). The core walls (simbyok) are the basic infill between the timber columns. This is a highly plastic structural system of woven bamboo, millet or slatted mats are fixed between the columns and covered with a plaster mixture of a clay and hay (30). These core walls are then finally finished with paper or cloth on the interior face, while on the exterior it is left bare exposing the timber substructure or lime washed as finish.

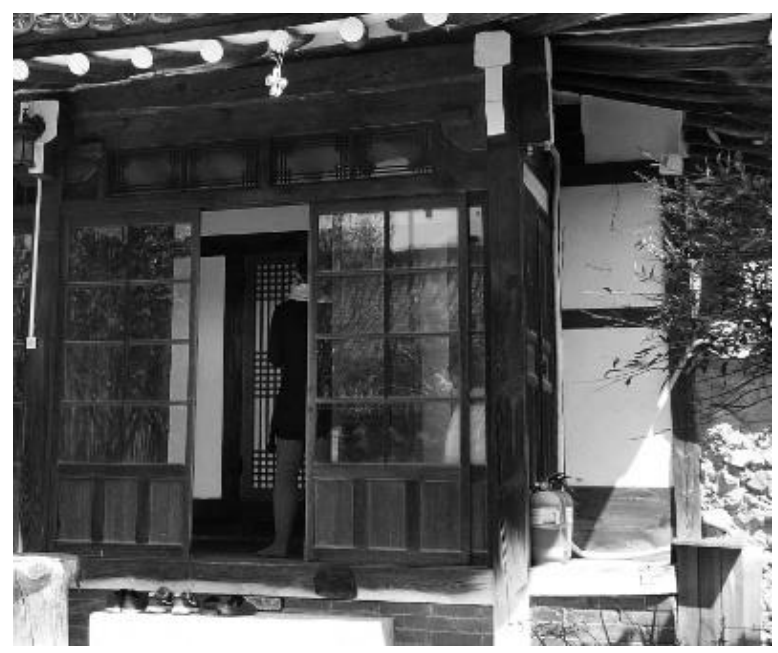

Figures 6. Screen walls and panels at a guesthouse currently in use in Hwangnamdong, Gyeongju, South Korea

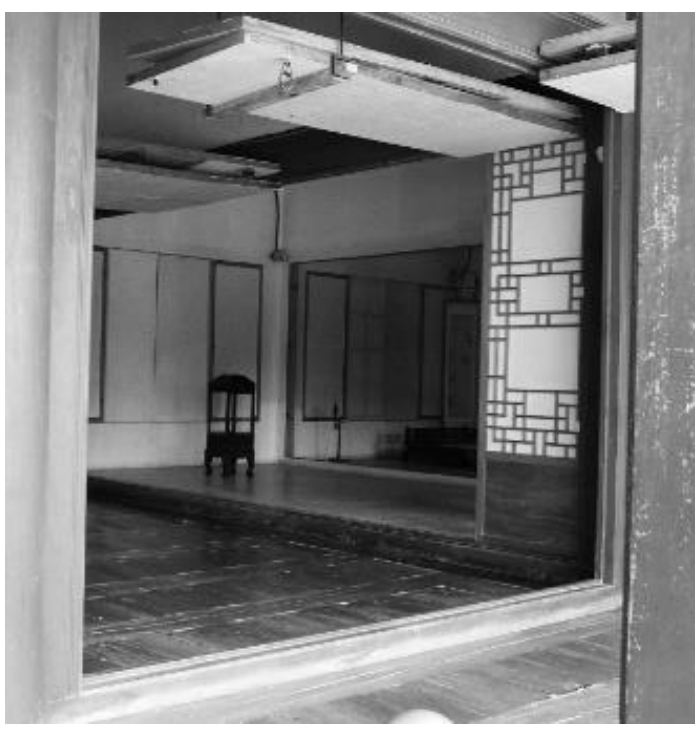

Figures 7. Screen walls and panels at Gyeonbuk Palace, Seoul

Large sections of walls are omitted to accommodate pocket sliding doors, hinge and sliding doors and tilting panels. All these panels, doors and windows are referred to as ch'angho or ch'angmun in the Hangeul (30) and typically do not differentiate in construction, material or finish. All openings are finished with similar materials as the interior walls; becoming in turn extensions of the wall plane. Instead, therefore, functioning as separate openings, these openings are articulated and scaled as flexible wall planes. 
All screens typically function as layered systems allowing for varying degrees of insulation, privacy and transparency [30]. Each layer differing in finish and structure; ranges from removable highly insulating timber and cotton panels, multiple layered paper screens to lightweight transparent paper screens. These can be added or removed as the need arises.

\subsection{Furniture as flexible armature expressing the building ideal}

The floor and furniture pieces are the final armature enabling multi-functionality and flexibility in the Hanoak house. As chairs aren't used the floor becomes a furniture piece - accommodating sleeping, sitting, resting and studying. Being in direct contact with the user, the floor becomes spatially intimate resulting in its careful articulation in terms of texture and finish [30].

All the rooms in the Hanoak house follow the Confucian principle of sparseness and reserved luxury for furniture use. All furniture especially - in the main hall (taech'ong) - are removable after a specific ritual or activity is conducted [30]. Most furniture pieces are, subsequently, designed according to basic principles of collapsibility, flexibility, are stackable or provide extensive storage - leaving the floor free to accommodate the variety of functions as needed [10, 30]. These furniture pieces have also been adapted to be smaller in overall size and much lower; only functioning at about 400$600 \mathrm{~mm}$ above the floor level, using the floor as complimenting armature.

\section{DISCUSSION}

The analysis of the Hanoak house typology reveals a series of architectural solutions that are:

a) highly responsive to diverse climatic conditions,

b) allow for a variety of functions to be performed within these spaces,

c) enable and exploit a series of multivalent building components to both increase resource efficiency and comfort,

d) and allow for the adaptation and development of the homesteads over time.

This high level of flexibility provides important lessons for the modern spatial and technological articulation of architectural solutions.

While a number of studies identify adaptation agency as being an important parameter in flexible architectural solutions (19), this study concurs with the findings of Till \& Schneider (2005) that in addition to a number of social-cultural and management aspects, tangible spatial and technological factors can also contribute to flexibility. This highlights the role of the designer when planning and executing certain design commissions. Learning from historic precedents that achieved high levels of flexibility and adaptive capacity using local materials, with limited advanced technologies and highly accessible technological solutions can inform these design decisions - specifically in contexts of limited surplus resources. From the analysis a series of principles can be derived as discussed in section 7.1.

\subsection{Principles and elements of adaptability}

Hanoak houses, as precedents, clearly depict elements of flexibility which have been successfully used in historic Korean homes. This level of flexibility was achieved on a human scale, while being very robust at the same time. From these precedents a series of principles can be drawn informing, in turn, flexibility in modern architecture:

a) Architectural flexibility and adaptability must be implemented as a holistic system, that is spatially, tectonically, functionally, and systemically flexible to ensure optimum indoor comfort. Instead of using only one aspect of flexibility within an inflexible whole, these precedents integrate this principle from the overall building design through to the use and choice of furniture.

b) Use smaller flexible and adaptable elements which are layered, inter-dependant and modular; this leads to an overall flexible, yet robust, architectural solution. On an individual basis none of these elements are highly complex or exceptionally adaptable, yet the complete system allows for countless possibilities.

c) Flexible elements cannot be added later as an add-on to an intervention. From the inception of the design an adaptable system must be utilised.

d) Flexibility is limited and functions within a robust unchanging framework; therefore, a fixed structure accommodates adaptability. These systems, in varied scales, need to be identified and treated as such. As shown in the study, using two different inflexible floor systems simultaneously greatly improve the overall adaptability of the system.

e) Hanoak houses generally exhibit similar characteristics and follow concurrent principles, yet regionally these houses have been adapted to specific contexts and microclimates $(29,30)$. This required an intimate knowledge and understanding of the context and climate. As much as adaptable systems are interchangeable and transferable amid different contexts and cultures, these systems must be adapted to accommodate the particularities of the local culture and context.

f) Flexible systems need not be highly developed digital technology, these can also be simple, robust products. Using building elements and technology constructed by local craftsmen and working closely with the end-user, guarantee that the system is legible and ensures its adoption and maintenance.

g) Use small, anthropomorphically scaled rooms with large opening or planes to ensure continued flexibility. The room sizes of Hanoak houses are specifically anthropocentrically scaled, limiting all indoor spaces to the smallest possible size. This allows for spaces to become very intimate, improves temperature control and minimises material use. Using the flexible partitioning, along with the undefined nature of the main hall allows the smaller rooms to accommodate a variety of different practices and rituals.

\section{CONCLUSION}

This study identified the architectural elements, spatial definition and technological articulation of Korean Hanoak architecture to highlight the value of vernacular knowledge to modern architectural applications. The findings contribute to our understanding of improving the flexibility of architectural solutions. This analysis of Korean vernacular architecture reveals that flexible spatial and technological systems were used extensively in multiple existing and historic precedents. 
As contribution to the architectural discourse this study considers a vernacular architectural typology and how its use of technology and space can inform modern architectural interventions. It specifically develops a set of principles that can inform new built solutions and highlights how flexibility can be enabled on an architectural scale. Ultimately, as revealed in these vernacular precedents, the use of local, comprehensible technologies can inform flexible architectural solutions in poorer resource constrained contexts to maximise it functional use and improve user comfort under rapidly changing conditions.

The analysis identified seven principles to inform flexible and adaptable architectural interventions. To effectively achieve an adaptable and flexible solution, the architecture must be developed following a holistic design approach that employs flexible strategies from the project inception. In addition, using small, simple flexible elements in a systemic inter-dependant manner is critical to achieve a flexible endproduct. Importantly, all flexible entities must function within inflexible frameworks - the two systems must be developed concurrently.

The survey of the examples reveals that simple, local and low technological solutions are appropriate in flexible systems. These are more robust, comprehensible by the end-user and can be maintained during the building's lifecycle. Finally, flexibility and spatial configuration are interdependent, using smaller anthropocentric ergonomic indoor spaces facilitate flexibility; yet, simultaneously, flexibility enables the use of smaller indoor spaces.

As further research these principles can be tested and documented in new owner-built architectural solutions. This can enable the identification of specific local technologies and solutions that enable flexibility within particular contexts.

\section{ACKNOWLEDGMENT}

The author would like to thank the reviewers for their constructive feedback and suggestions to improve the article.

\section{REFERENCES}

[1] Singh, M.K., Mahapatra, S., Atreya, S.K. (2011). Solar passive features in vernacular architecture of North-East India. Solar Energy, 85(9): 2011-2022. https://doi.org/10.1016/j.solener.2011.05.009

[2] Chandel, S.S., Sharma, V., Marwah, B.M. (2016). Review of energy efficient features in vernacular architecture for improving indoor thermal comfort conditions. Renewable and Sustainable Energy Reviews, 65:

459-477. http://dx.doi.org/10.1016/j.rser.2016.07.038

[3] Intergovernmental Panel on Climate Change. (2018). Global warming of $1.5^{\circ} \mathrm{C}$ : An IPCC special report on the impacts of global warming of $1.5^{\circ} \mathrm{C}$ above pre-industrial levels and related global greenhouse gas emission pathways, in the context of strengthening the global response to the threat of climate change, sustainable development, and efforts to eradicate poverty. Intergovernmental Panel on Climate Change.

[4] Laukkonen, J., Blanco, P.K., Lenhart, J., Keiner, M., Cavric, B., Kinuthia-Njenga, C. (2009). Combining climate change adaptation and mitigation measures at the local level. Habitat International, 33(3): 287-292. https://doi.org/10.1016/j.habitatint.2008.10.003

[5] Walker, B., Salt, D. (2006). Resilience Thinking: Sustaining Ecosystems and People in a Changing World. Island Press.

[6] Crichton, D., Nicol, F., Roaf, S. (2009). Adapting Buildings and Cities for Climate Change. Routledge, Oxford.

[7] Tan, G. (2011). The Open and Adaptive Tradition: Applying the concepts of open building and multipurpose design in traditional Chinese vernacular architecture. Journal of Asian Architecture and Building Engineering, $\quad 10(1)$ : 7-14. https://doi.org/10.3130/jaabe.10.7

[8] Till, J., Schneider, T. (2005). Flexible housing: the means to the end. ARQ: Architectural Research Quarterly, 9(34):

https://doi.org/10.1017/S1359135505000345

287-296.

[9] Fernandes, J., Mateus, R., Bragança, L., Correia da Silva, J.J. (2015). Portuguese vernacular architecture: the contribution of vernacular materials and design approaches for sustainable construction. Architectural Science Review, 58(4): 324-336. https://doi.org/10.1080/00038628.2014.974019

[10] Kim, S., Jia, B. (2012). Flexible building and construction systems in traditional Korean architecture. Open House International, 37(3): 16-27. https://doi.org/10.1108/OHI-03-2012-B0003

[11] Archer, E., Engelbrecht, F., Hänsler, A., Landman, W., Tadross, M., Helmschrot, J. (2018). Seasonal prediction and regional climate projections for southern Africa. In: Revermann R., Krewenka K.M., Schmiedel U., Olwoch J.M., Helmschrot J., Jürgens N. (eds) Climate change and adaptive land management in southern Africa assessments, changes, challenges, and solutions Klaus Hess Publishers, Göttingen \& Windhoek, 14-21.

[12] Intergovernmental Panel on Climate Change. (2014). Synthesis Report. Contribution of Working Groups I, II and III to the Fifth Assessment Report of the Intergovernmental Panel on Climate Change. Cambridge University Press, New York.

[13] Herrera, M., Natarajan, S., Coley, D.A., Kershaw, T., Ramallo-González, A.P., Eames, M., Wood, M. (2017). A review of current and future weather data for building simulation. Building Services Engineering Research and Technology, 38(5): https://doi.org/10.1177/0143624417705937

[14] Smit, B., Wandel, J. (2006). Adaptation, adaptive capacity and vulnerability. Global Environmental Change, 16(3): 282-292. https://doi.org/10.1016/j.gloenvcha.2006.03.008

[15] O'Brien, G., O'keefe, P. (2013). Managing Adaptation to Climate Risk: Beyond Fragmented Responses. Routledge, Oxon.

[16] Pelling, M., O’Brien, K., Matyas, D. (2015). Adaptation and transformation. Climatic Change, 133(1): 113-127. https://doi.org/10.1007/s10584-014-1303-0

[17] Campbell, A.D. (2017). Lay designers: Grassroots innovation for appropriate change. Design Issues, 33(1): 30-47. https://doi.org/10.1162/DESI_a_00424

[18] Leichenko, R. (2011). Climate change and urban resilience. Current Opinion in Environmental Sustainability, $\quad 3(3)$ : $\quad 164-168$. https://doi.org/10.1016/j.cosust.2010.12.014 
[19] Dipasquale, L., Kisa Ovalı, P., Mecca, S., Özel, B. (2014). Resilience of Vernacular Architecture. Versus: Heritage for Tomorrow; Firenze University Press: Florence, Italy, 65-73.

[20] Folke, C., Carpenter, S.R., Walker, B., Scheffer, M., Chapin, T., Rockström, J. (2010). Resilience thinking: integrating resilience, adaptability and transformability. Ecology and Society, 15(4): 20.

[21] Carter, J.G., Cavan, G., Connelly, A., Guy, S., Handley, J., Kazmierczak, A. (2015). Climate change and the city: Building capacity for urban adaptation. Progress in Planning, 95: https://doi.org/10.1016/j.progress.2013.08.001

[22] Heinrichs, D., Krellenberg, K., Fragkias, M. (2013). Urban responses to climate change: Theories and governance practice in cities of the global south. International Journal of Urban and Regional Research, 37(6): $\quad 1865-1878$. https://doi.org/10.1111/14682427.12031

[23] Carter, J.G. (2011). Climate change adaptation in European cities. Current Opinion in Environmental Sustainability, 3(3): 193-198. https://doi.org/10.1016/j.cosust.2010.12.015

[24] Lwasa, S. (2010). Adapting urban areas in Africa to climate change: the case of Kampala. Current Opinion in Environmental Sustainability, 2(3): 166-171. https://doi.org/10.1016/j.cosust.2010.06.009

[25] Cartwright, A., Blignaut, J., De Wit, M., Goldberg, K., Mander, M., O’Donoghue, S., Roberts, D. (2013).
Economics of climate change adaptation at the local scale under conditions of uncertainty and resource constraints: The case of Durban, South Africa. Environment and Urbanization, 25(1): 139-156. https://doi.org/10.1177/0956247813477814

[26] Shahin, H.S.M. (2019). Adaptive building envelopes of multistory buildings as an example of high performance building skins. Alexandria Engineering Journal, 58(1): 345-352. https://doi.org/10.1016/j.aej.2018.11.013

[27] Groat, L.N., Wang, D. (2013). Architectural Research Methods. John Wiley \& Sons, New Jersey.

[28] Yun, J. (2015). A study of the planning characteristics of Neowa houses applicable in contemporary housing plans. Journal of the Korean Housing Association, 26(2): 81-88. https://doi.org/10.6107/JKHA.2015.26.2.081

[29] Kim, D. (2013) History of Korean Architecture. Edited by Gregory A. Tisher and translated by Jong-hyun Lim. Suwon: University of Kyonggi Press.

[30] Choi, J.S., Chumn, H.J., Hong, H.O., Kang, S.J., Kim, D.N., Min, C.H. (1999). Hanoak: Traditional Korean Homes. Hollym Corp, Seoul.

[31] Korean Meteorological Administration. (2014). Climate of Korea. 2014. http://web.kma.go.kr/eng/biz/climate_01.jsp, accessed on May 28, 2014

[32] Han, Y.W., Kim, D.B. (2007). Dongwaldo - Painting of the Eastern Palace. Changdeokgun and Changgeyongung. Hyonghung, Paju. ISBN: 978-89-5872-041-6 02910. 